\title{
Convolutive Blind Source Separation based on Wavelet De-noising
}

\author{
Hong-bin Zhang \\ Faculty of Computer and Communication Engineering \\ Huaian College of Information Technology \\ Huai'an ,China \\ e-mail: zhb615508@sohu.com
}

\author{
Peng-fei Xu \\ Faculty of Computer and Communication Engineering \\ Huaian College of Information Technology \\ Huai'an ,China \\ e-mail:jionad@126.com
}

\begin{abstract}
The paper discusses the time-domain blind seperation applied to communication signals, using an ICA algorithm EFICA together with a wavelet de-noising processing method. In the Blind source separation system, regardless of the mixed signals and separated signals, noise pollution occurs frequently, it increases the complexity of BSS and the difficulty of dealing with the aftermath. So an automatic method of and wavelet de-noising processing is proposed finally. It yields good results in the experiment and improves the performance of BSS system.
\end{abstract}

Keywords: Convolutive mixture; Time-Domain Blind Source Separation; Communication Signals; Wavelet Denoising

\section{INTRODUCTION}

Blind signal separation(BSS), also known as blind source separation, is the separation of a set of signals from a set of mixed signals, without the aid of information (or with very little information) about the source signals or the mixing process. It has been intensively investigated in the recent two decades. This problem is important for several applications, such as communications, biomedical engineering and blind audio source separation. In general, the sources are assumed to be independent or at least decorrelated.

First we introduce the basic model of convolutive mixtures. a mixture of $n$ source signals $s(k)=\left[s_{1}(n), s_{2}(n), \ldots, s_{n}(n)\right]^{T}$ are received at an array of $m$ sensors. The received signals are denoted $x(k)=\left[x_{1}(n), x_{2}(n), \ldots, x_{m}(n)\right]^{T}$. In many real-world applications the sources are said to be convolutively (or dynamically) mixed. The noise-free convolutive mixing model is given as

$$
\begin{aligned}
x(k) & =\sum_{p=0}^{P-1} H(p) s(k-p) \\
\text { where } H(p) & =\left[\begin{array}{ccc}
h_{11, p} & \cdots & h_{1 n, p} \\
\vdots & \ddots & \vdots \\
h_{m 1, p} & \cdots & h_{m n, p}
\end{array}\right] \text { is the } m \times n \text { FIR- }
\end{aligned}
$$

filter mixing matrix of length $P . h_{i j}$ is impulse responses between the $j$ 'th source signal and the $i$ 'th sensor. In the real environment, the source signals pass through the transmission channel with different time delays before arriving at the receiver.

The objective is to design a causal, stable MIMO(Multiple-Input Multiple-Out-put) separation filter matrix $W(q)$ of length $Q$ with outputs given as:

$$
y(k)=\sum_{q=0}^{Q-1} W(q) x(k-q)
$$

which is the estimation signal of source $s(k)$.

Here, we address the blind separation of communication signals by means of a powerful ICA algorithm EFICA [1] as a decomposition method for the time-domain separation. In the Blind source separation system, regardless of the mixed signals and separated signals, it often appears situation of noise pollution , this increases the complexity of BSS and the difficulty of dealing with the aftermath. thus, an automatic method of and wavelet de-noising processing is proposed too, using the 'mini-maxi' soft-threshold model, wavelet decomposition is performed at level 5 for the separated noisy communication signal. The method above is demonstrated by the experiments for communication signals with same carrier frequencies and modulation.

The paper is organized as follows. In Section 2 and 3, the steps of BSS and wavelet denoising are introduced briefly. Section 4 gives some experimental results to demonstrate the effectiveness of the method for communication signals in time-domain with conclusion given in the last section.

\section{THE STEPS OF BSS IN THE TIME-DOMAIN}

The goal of convolutive BSS is typically to estimate the contributions of all sources in each observation, Under the assumption that the souce signals are independent and identically distributed (i.i.d.) and nongaussian sequences, the number of source signals is set to $N$, the number of observation signals is set to $P$, the general form of BSS in the paper can be described as follows.

(1) zero-mean

Make sure the mixed signals

$$
x(n)=\left[x_{1}(n), x_{2}(n), \ldots, x_{P}(n)\right]^{T} \text { is zero-mean }
$$

(2) convolutive sphering

At any time $n$, we consider the column vector 


$$
\tilde{x}(n)=\left[x_{1}(n+R), \ldots, x_{1}(n-R), \cdots, x_{P}(n+R), \ldots, x_{P}(n-R)\right]^{T}
$$

which contains $M=(2 R+1) P$ entries. We derive the $M$ - entry column vector

$$
\begin{gathered}
x^{\prime}(n)=\left[x_{1}^{\prime}(n), \ldots, x^{\prime}{ }_{M}(n)\right]^{T} \text { defined as } \\
x^{\prime}(n)=B \tilde{x}(n)
\end{gathered}
$$

where $B$ is an $M \times M$ matrix

$$
E\left\{x_{i}^{\prime}(n) x^{\prime}{ }_{j}(n)\right\}=\delta_{i j}, \quad \forall i, j \in\{1, \ldots, M\}
$$

(3) and (4) shows that the signals $x_{i}^{\prime}(n)$ are convolutive mixtures of the $x_{i}(n)$.then (5) means that the signals $x_{i}^{\prime}(n)$ are created so as to have unit variances and to be mutually uncorrelated, which may be seen as a spatiotemporal whitening and normalization of the observations, (4) is actually a whitening process, $B$ is equivalent to a whitening matrix.

(3) fixed-point algorithm[2]

This assumes that the signal extraction is:

$$
y(n)=W \cdot x^{\prime}(n)=\sum_{m=1}^{M} \omega_{m} x_{m}^{\prime}(n)
$$

where $W$ is an $M$-entry extended column vector of extraction coefficients $\omega_{m}$, together with (4), it yields a convolutive combination $y(n)=\left[y_{1}(n), \ldots, y_{M}(n)\right]^{T}$ of the observations.

(4) component grouping

This step is based on the premise [3] : The $M$ independent components of $y(n)=\left[y_{1}(n), \ldots, y_{M}(n)\right]^{T}$ can be clustered into $N$ groups, each group contains only one source of information.

Here, a distance measure is defined to express the relationship between the independent components ,

$$
D_{i j}=\left\|y_{j}-C_{i}\left(C_{i}^{T} C_{i}\right)^{-1} C_{i}^{T} y_{j}\right\|^{2}
$$

where the matrix

$$
\begin{gathered}
C_{i}=\left[D^{-R} y_{i}, D^{-R+1} y_{i}, \ldots, D^{R-1} y_{i}, D^{R} y_{i}\right] \text {, the } \\
\text { vector } y_{i}=\left[\begin{array}{c}
y_{i}(R+1) \\
y_{i}(R+2) \\
\vdots \\
y_{i}(L-R-1) \\
y_{i}(L-R)
\end{array}\right] \text {, the vector }
\end{gathered}
$$

$$
D^{k} y_{i}=\left[\begin{array}{c}
y_{i}(R+k+1) \\
y_{i}(R+k+2) \\
\vdots \\
y_{i}(L+k-R-1) \\
y_{i}(L+k-R)
\end{array}\right] \text {, }
$$

$k=-R, \ldots, R . i=1,2, \ldots, P, L$ is sampling length.

Calculate $D_{i j}$ and cluster the components by hierarchical clustering method[4] .

(5) reconstruct signal

Once the independent components are clustered to $N$ groups, each group(say $j$-th) is used for reconstruction of the corresponding original signal. That is to say, after clustering the receive signals for one receiver, the $j$-th group is extracted. then we do it again for each receiver and constructively sum the delayed versions of the signal on each receiver separately.

Here, $y^{(j)}(n)$ is the clustered group of $y(n)$, where $j=1, \ldots, N$. For each group, we use the following formula to reconstruct $x^{(j)}(n)$, the signals are delayed versions of the $j$-th source received on all receivers;

$$
x^{\prime(j)}(n)=W^{-1} y^{(j)}(n)
$$

We constructively sum the delayed versions of the signal on each receiver separately, so that the contribution of the source on the $i$-th receiver is

$$
\breve{S}_{i}^{(j)}(n)=\sum_{p=1}^{2 R+1} x_{(i-1)(2 R+1)+p}^{(j)}(n+p-1)
$$

According to the method above, all of the $N$ input signals are reconstructed finally.

(6) wavelet denosing

Wavelet denoising analysis in section 3 is introduced to denoise the separated signal, denoising is a filtering process of reducing the high-frequency noise and reconstructing the low-frequency signal, or the details.

\section{WAVELET DENOISING}

In the processes of transmitting and receiving signal, all kinds of noise are accumulated, the separated communication signal often carry a lot of noise. In order to improve the performance of blind source separation system, it is necessary to denoise the separated signal. Wavelets have an important application in signal denoising, Here we use wavelet de-noising. The method of wavelet threshold de-noising is based on the principle of the multiresolution analysis. The discrete detail coefficients and the discrete approximation coefficients can be obtained by a multi-level wavelet decompose. It has been proved[5] that the variances and amplitudes of the details of the white noise at the various levels decrease regularly as the level increase. On the other hand, the amplitude and variances of wavelet transform of the available signal are not related to the change of scale. According to the properties of wavelet transform of 
the noise and the available signal, we can weaken, and even remove noise.

We denoise the separated signal by using wedn function in Matlab, wden performs an automatic de-noising process of a one-dimensional signal using wavelets.

$\mathrm{XD}=$ wden $(\mathrm{X}, \mathrm{TPTR}, \mathrm{SORH}, \mathrm{SCAL}, \mathrm{N}$, 'wname') returns a de-noised version $\mathrm{XD}$ of input signal $\mathrm{X}$ obtained by thresholding the wavelet coefficients. TPTR string is threshold selection rule, here we use 'minimaxi' for minimax thresholding. That is, TPTR='minimaxi'. SORH ('s' or 'h') is for soft or hard thresholding, SCAL defines multiplicative threshold rescaling, here we use 'mln' for rescaling done using level dependent estimation of level noise. Wavelet decomposition is performed at level $\mathrm{N}$ and 'wname' is a string containing the name of desired orthogonal wavelet. Here, $\mathrm{N}=5$, wname=sym5. In this paper, the wavelet denoising code are as follows:

$\mathrm{Xd1}=$ wden(U1, 'minimaxi', 's', 'mln', lev, 'sym5'),

$\mathrm{Xd} 2=$ wden(U2, 'minimaxi', 's', 'mln', lev, 'sym5'),

Here, U1 and U2 are two separated signal which is seriously affected by noise pollution, $\mathrm{Xd}$ and $\mathrm{Xd} 2$ are denoised version of input signal obtained by thresholding the wavelet coefficients.

\section{EXPERIMENTAL RESULTS}

In order to verify the effectiveness of the above method for convolutive BSS of communication signal, the research simulations are done using matlab software. We assume that the source signals are QPSK signals with same carrier frequency $60 \mathrm{KHz}$. In order to facilitate the description, the number of source singals $n$ is set to 2, the number of sensors $m$ is also set to 2 . The sampling points is 200000, the length of mixing FIR-filter is 5, they pass through the AWGN (additive white Gauss noise) channel which have the SNR (Signal Noise Ratio) of 20 $\mathrm{dB}$. The mixing matrix $H(p)$ is a $2 \times 2$ random matrix , the elements are randomly generated subject to the uniform distribution in $[0,1]$.

Under the previous assumptions, the figure 1 shows the waveform of two source signals, the figure 2 shows the waveform of two signals through the AGWN channel, From figure 1 to figure 5 , the abscissa is the number of sampling points, the longitudinal coordinate is the amplitude of waveform.

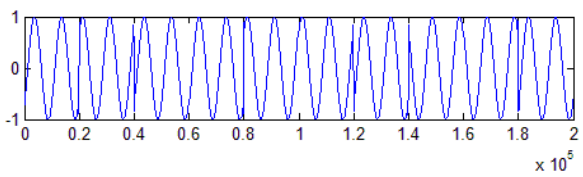

(a) waveform of S1

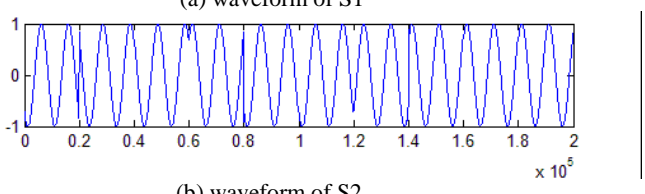

(b) waveform of S2

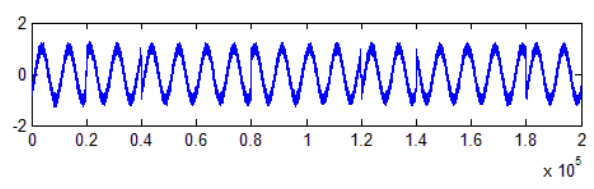

(a) waveform of S1 through AWGN channel

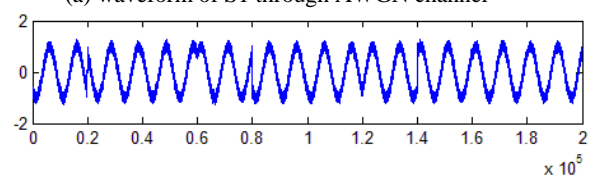

(b) waveform of S2 through AWGN channel

Figure 2. waveform of two source signals which are transmitted through an AWGN channel

The figure 3 shows the waveform of two observation signals and the figure 4 shows the waveform of two separated signals.
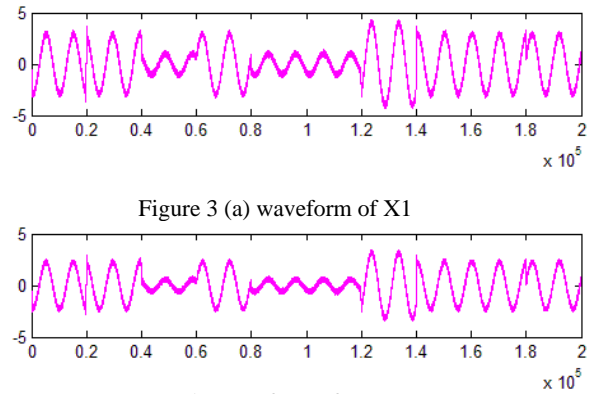

(b) waveform of $\mathrm{X} 2$

Figure 3. waveform of two observed signals for convolutive mixtures

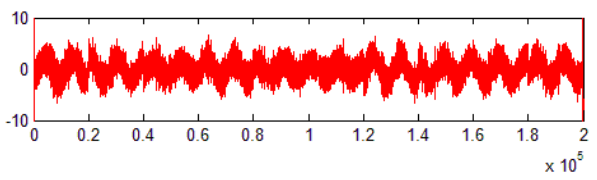

(a) waveform of U1

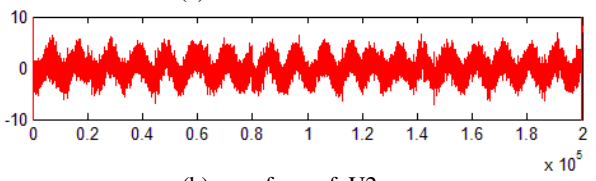

(b) waveform of U2

Figure 4. waveform of two separated signals

From the figure 4 , we can see the noise of separated signal is serious. In order to recognize signal better, we need denoise the separated signal by the wavelet denoising method described in the section 3 . the results shows in figure 5 and table 1 . we record denoised separation signal as $\mathrm{Xd} 1$ and $\mathrm{Xd} 2$. The restoration of the original signal is good after de-noising the signal. It can improve the performance of BSS system.

Figure 1. waveform of two source signals 


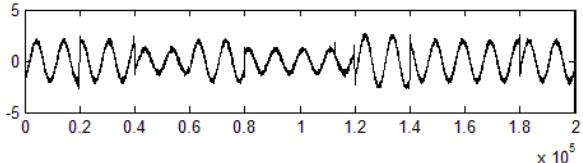

(a) waveform of $\mathrm{Xd} 1$

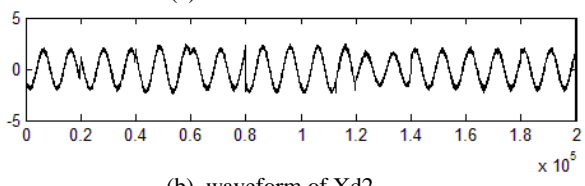

(b) waveform of $\mathrm{Xd} 2$

Figure 5 waveform of two separated signals after denoising

From the table 1 ,we can see the Correlation Coefficient (CC) of the source signals and the separated signals, the CC of $\mathrm{S} 1$ and $\mathrm{Xd} 1$ close to 1 , the $\mathrm{CC}$ of $\mathrm{S} 2$ and $\mathrm{Xd} 2$ close to 1 too,that means separation effect is good.

TABLE I. CORRELATION COEFFICIENT OF THE SOURCE SIGNALS AND THE SEPARATED SIGNALS

\begin{tabular}{|c|c|c|}
\hline source signal & S1 & S2 \\
\hline separated signat & $\underline{0.8558}$ & 0.1141 \\
\hline $\mathrm{Xd} 1$ & 0.1113 & $\underline{0.9850}$ \\
\hline $\mathrm{Xd} 2$ &
\end{tabular}

In addition, we can use the constellation to view the phase information of separated signal .

Figure 6 shows the constellation of observation signal, we can see the constellation points has been severely disrupted and can't find the correct position of the constellation points.

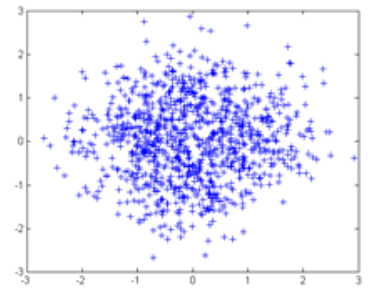

(a) constellation diagram of $\mathrm{X} 1$ Figure 6. Two constellation diagram of observed signals

Figure 7 shows the constellation of separation signal, the red dot is the original QPSK constellation diagram . the composition of blue ' + ' is the constellation of separated signal.

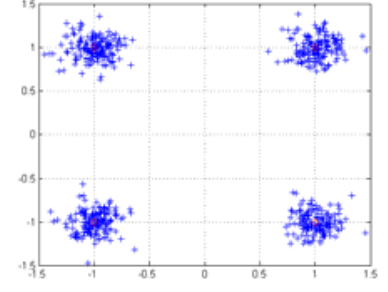

(a) constellation diagram of $\mathrm{Xd1}$

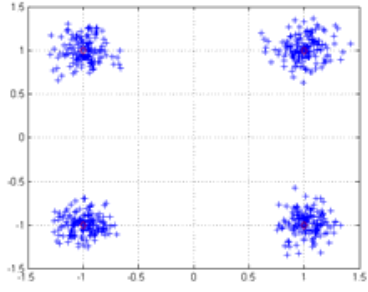

(b) constellation diagram of $\mathrm{Xd} 2$
From the above figures and table, we see that the method in the paper can separate source signals from the observation signals effectively.

\section{CONCLUSIONS}

In this paper, a method for convolutive blind separation for communication sources is introduced. The method works in time-domain, and it is based on the recently very successful algorithm EFICA for Independent Component Analysis, which is an enhanced version of more famous FastICA. In addition, an automatic method of wavelet denoising processing is proposed, using the 'mini-maxi' softthreshold model, wavelet decomposition is performed at level 5 for the noisy separated communication signal, it can improve the performance of BSS system, and this is confirmed in the experiment for communication signals with same carrier frequencies and modulation. The method is also suitable for other forms of signal for convolutive mixtures, such as the spectrum aliasing signal and signals of different frequency, etc.

\section{REFERENCES}

[1] Koldovsky Z., Tichavsky P., Oja E. Efficient variant of algorithm. fastICA for independent component analysis attaining the CramerRao lower bound. IEEE Tr. Neural Networks, 17 (2006) 1265-1277.

[2] Thomas, J., Deville, Y., Hosseini, S.: “Time-domain Fast Fixed-Point Algorithms for Convolutive ICA”, IEEE Signal Processing Letters, 13(4) (2006) 228-231.

[3] Z. Koldovský and P. Tichavský. Time-Domain Blind Audio Source Separation Using Advanced ICA Methods [J].Proceedings of 8th Annual Conference of the International Speech Communication Association (Interspeech 2007).August 2007, pp. 846-849.

[4] Späth H. cluster analysis algorithms for data reduction and classification of objects. Chichester,Ellis Horwood .1980.

[5] Dai-fei Guo, A study of Wavelet thresholding de-noising, IEEE Proceedings of ICSP, pp.329-332, 2000. 